\title{
“One-Piece" Immediate-Load Post-Extraction Implant In Maxillary Central Incisor
}

\author{
Abdulgani Azzaldeen, Chlorokostas Georges, Abu-Hussein Muhamad*
}

\begin{abstract}
This case report describes extraction of a fractured left maxillary central incisor tooth, followed by immediate placement of an one-piece implant in the prepared socket and temporization by a bonded restoration. Materials And Methods: The tooth was extracted with minimal hard and soft tissue trauma and without flap reflection. The socket was prepared to the required depth and a Implant was inserted.

Results: The atraumatic operating technique and the immediate insertion of the one-piece Implant resulted in the preservation of the hard and soft tissues at the extraction site.

Conclusion: The "One-piece" dental implant and provisional restoration provided the patient with immediate esthetics, function, comfort and most importantly preservation of tissues. The one-piece implant design resulted in a high cumulative implant survival rate and beneficial marginal bone levels.
\end{abstract}

Keywords: Immediate placement, Immediate temporization; One-piece implant; Primary stability

\section{Introduction}

Osseointegrated dental implants have traditionally been placed in accordance with the Brenemark's two stage protocol. Implants were submerged and left to heal for a period of 3 to 4 months in mandible and 6 to 8 months in maxilla.[1] This meant that patients had to wait a significant time before prosthesis placement and often had to wear suboptimal provisional prostheses. Initial attempts to load the implant earlier were associated with increased failure rates.[2,3] But with the many advances being made in the field of implantology, implants can now be successfully loaded early or immediately in selected cases.[4]

Immediate replacement of single tooth by provisional restoration of dental implants is a procedure of growing interest among clinicians worldwide. Single tooth loss is probably the most common indication for implant placement.[5] The loss of a single tooth is a traumatic experience for many patients and early/immediate implant loading is therefore an attractive treatment option. However, single teeth replaced by implants in the aesthetic zone are one of the most challenging situations facing a clinician, even when using a two-stage implant protocol.[6] Careful assessment thus must be made of mucosal and bone volumes for optimum implant aesthetics.[6,7]

Immediate implant placement allow advantages over conventional approach such as reduction in the number of surgical procedures and hence the treatment time required,[1] ideal axial orientation of the implant,[2-4] preservation of the bone at the extraction site and optimal soft-tissue aesthetics,[2,4] significantly reduced period of wearing of an interim prosthesis.[4] Studies showing high success rates of immediately placed implants have made this protocol more favorable for implantologists.[5,7]

However, this protocol has been associated with shortcomings like ideal modality for the treatment of marginal voids. These are the gap present between the implant body and the wall of the bony socket. The procedure is also technically more demanding. Although, implant placement in fresh extraction socket has been described previously, it is only recently that such clinical approach has gained popularity.[8] However, there is a lack of general consensus on the various aspects of immediate implant placement in an extraction socket, this article reviews the literature available on implants placed into the extraction socket and summarizes its clinical outcome.[6,7].Immediate loading of single-tooth implants in the esthetic zone can be the treatment of choice for both clinical and psychological reasons.[9]

Good evaluation and techniques to achieve good primary stability are important to ensure a successful implantation, an optimal situation in the buccal and muco-gingival areas, occlusion with support and a functional temporary restoration. Transversal forces that are exerted on the implant should be as minimal as possible. Highly esthetic results can be achieved.[9,10]

Implant-supported solutions such as a fixed temporary restoration and immediate loading contribute to the patients' quality of life at work and in their social lives. They meet the patients' expectations regarding esthetics and comfort. These advantages often outweigh the disadvantages and reduce treatment stress.[11,12]

The choice of the treatment depends on the indication. With an implant treatment, the aim is to offer the patient an efficient, quick and esthetic solution, provided that the patient agrees to an implantation. [13]In order to contribute to a successful implant treatment, the patient needs to participate actively. Patients should follow instructions regarding mastication during the first 6 weeks.[14] 
The following report describes a case of replacement of maxillary central incisor by an one-piece implant system with immediate provisional restoration.

\section{Case Report}

A 72-year-old female patient presented with a history of trauma and crown fracture at the cervical area of tooth 21 and requested an immediate solution. Clinical and radiological evaluation revealed adequate alveolar bone, absence of periapical pathology but fracture line was below the crest of alveolar bone and was limited to the tooth. It was decided to extract and place endosseous implant immediately and place a provisional restoration to avail the benefits like preservation of bone and emergence profile. After administering appropriate antibiotic and analgesic, induction of local anaesthesia was carried out using lignocaine with adrenaline. As preservation of alveolar bone is key to success of immediate implants, extraction of tooth has to be atraumatic, so using periotomes and small periosteal elevators the fragment was luxated without excessive enlargement of the socket, and using an innovative method where endodontic file was used to engage the canal wall and tooth fragment was slowly luxated and pulled out of the socket using. The sockets were debrided with curettes and a dental implant was planned $(3 \times 15 \mathrm{~mm})$. The drilling sequence was carried out without reflecting the flap to preserve the bone.

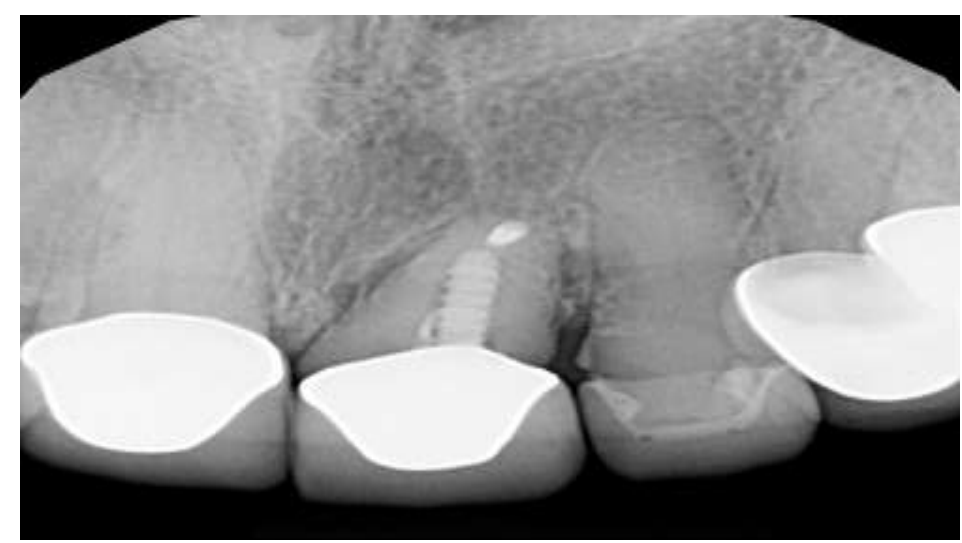

Fig.1 Pre-operative radiographic

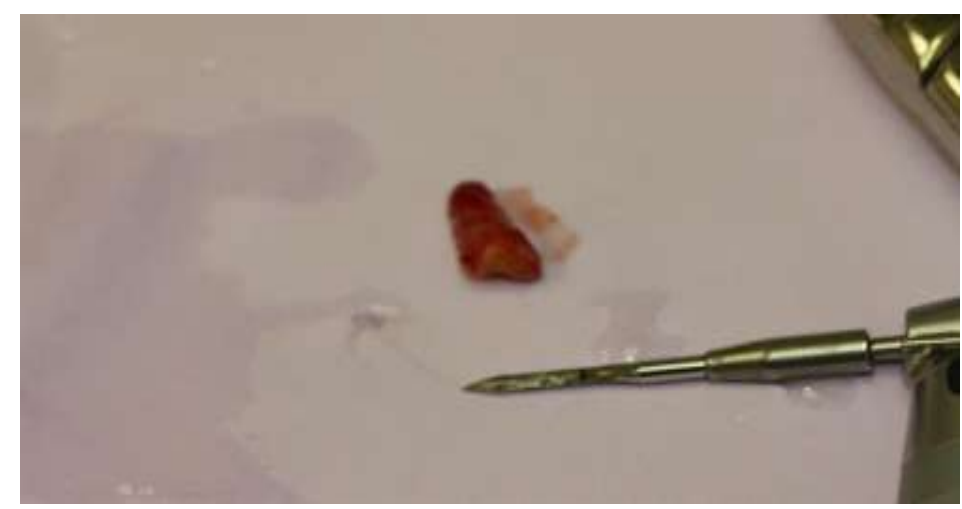

Fig.2Tooth extraction

According to the "keyhole" ("Schlósselloch") concept, the prepared bone cavity can be checked with a periodontal probe. After checking the bone cavity, the cavity is widened with bone expanders of $2 \mathrm{~mm}, 4 \mathrm{~mm}$, and $3 \mathrm{~mm}$ like an osteotome. Using the Torque Wrench, you can feel bone resistance and see which torque value can be reached. The periosteum is not injured, and there are just a few or even no followup surgeries. Depending on bone type, there are different preparation protocols to ensure good bone condensation and to reach a sufficient torque for placing a $\Phi 3.5 \mathrm{~mm}$ implant or when necessary a $\Phi 4.5 \mathrm{~mm}$ or $\Phi 5.5 \mathrm{~mm}$ implant. In this case, we placed a $\Phi 3 \mathrm{~mm}$ and $15 \mathrm{~mm}$ - long one-piece at a torque of $60 \mathrm{Ncm}$. 


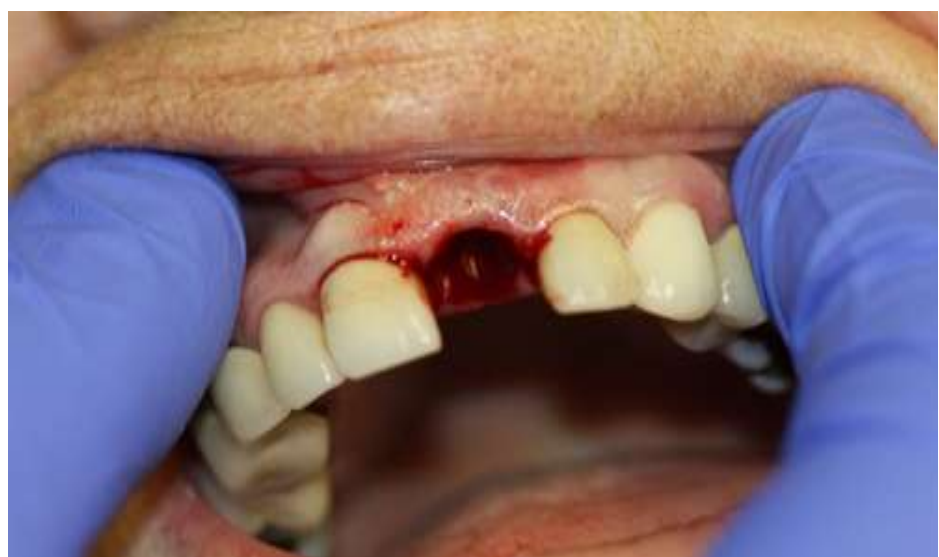

Fig.3Tooth extraction and the fresh socket

A temporary restoration was made. Excellent esthetics and proper function could be restored: contact points were checked under functional occlusion, and no anterior guiding elements were set on this tooth.Post operative instructions were given to the patient, and was asked to report after 1 week. The sutures were removed after 7 days and the patient received temporary acrylic crown bonded to the adjacent teeth with fibre-reinforced composite on the same day .

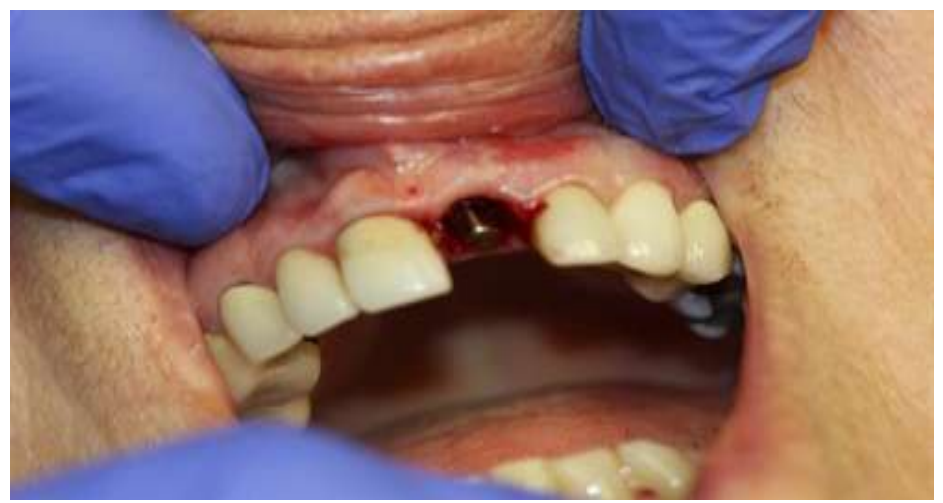

Fig.4 Tooth extraction and immediate implant placement in the fresh socket

\section{Discussion}

Missing teeth can cause loss of self-esteem and have an impact on social life. The implant-supported prosthesis can overcome these problems and has proved to be a significant addition to restorative dentistry.[5,6,7] This was one of the main reasons to consider an endosseous root- form implant. [ 6] One-piece design with no separate abutment screw was used in the present study since advantage of this design is increased strength, elimination of the risk of abutment screw loosening, and reduced crestal bone loss due to non-existence of microgap between the abutment and implant.[15]

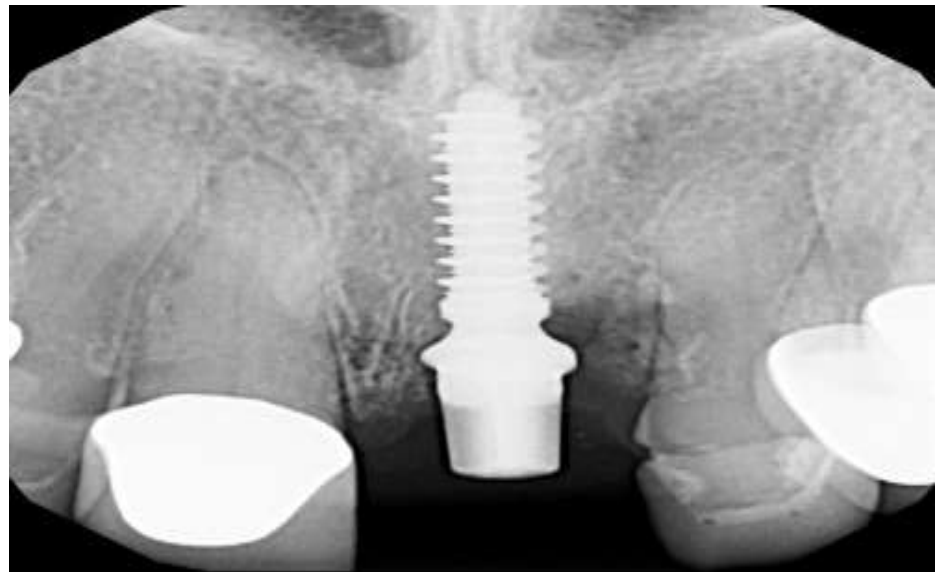

Fig.5 Radiographic images just after implant placement 
Immediate temporization of single tooth was considered since the site was in esthetic zone and there was need for ideal soft tissue drape. Immediate temporization in the form of non-functional immediate tooth carries certain advantages like fixed esthetic tooth replacement soon after surgery, no parafunctional forces from occlusion, no need to countersink the implant below the crestal bone, which reduces early bone loss, and excellent soft tissue emergence.[16]

Disadvantages of non-functional immediate teeth include micro movement of implant that can cause crestal bone loss and acrylic, which may become trapped under tissues or between implant and crestal bone. [17]This problem was reduced greatly as the implants used in present case report had the crest module larger in diameter than implant.[17,18]

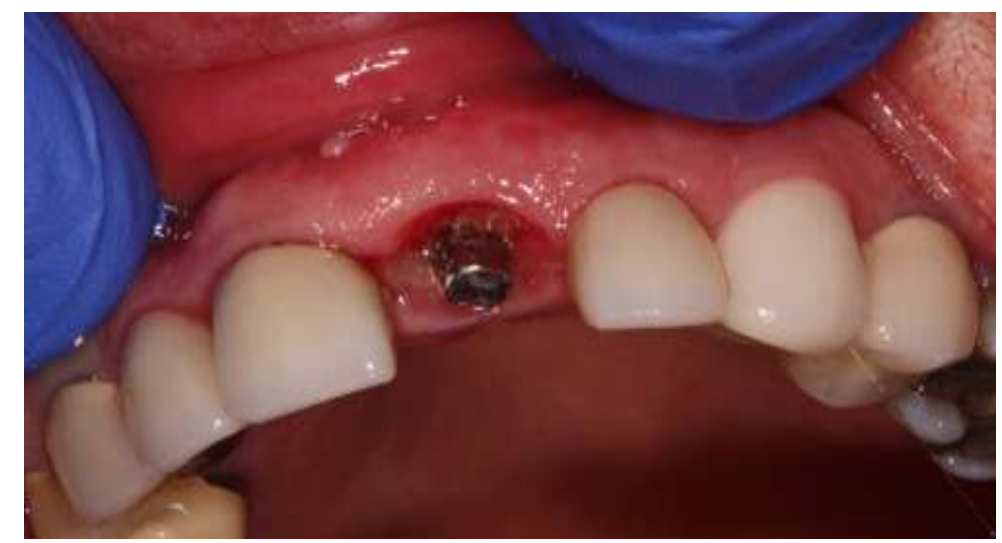

Fig.61 week post op

The primary disadvantage of immediate loading is the risk of implant failure or greater crestal loss around the healing implants. Overloading of the implant could be a factor for implant failure in immediate loading. Hence, we considered immediate temporization and not immediate functional loading.[19]emporary crowns had a positive impact on the soft tissue with respect to thepreservation of the papillae. Placement of implant at correct angulation is again very important.[16]The one-piece implant design enables undisturbed healing of the peri-implant soft tissue and avoids disruption of the soft tissue seal when placing the definitive prosthetic restoration.[15,16]

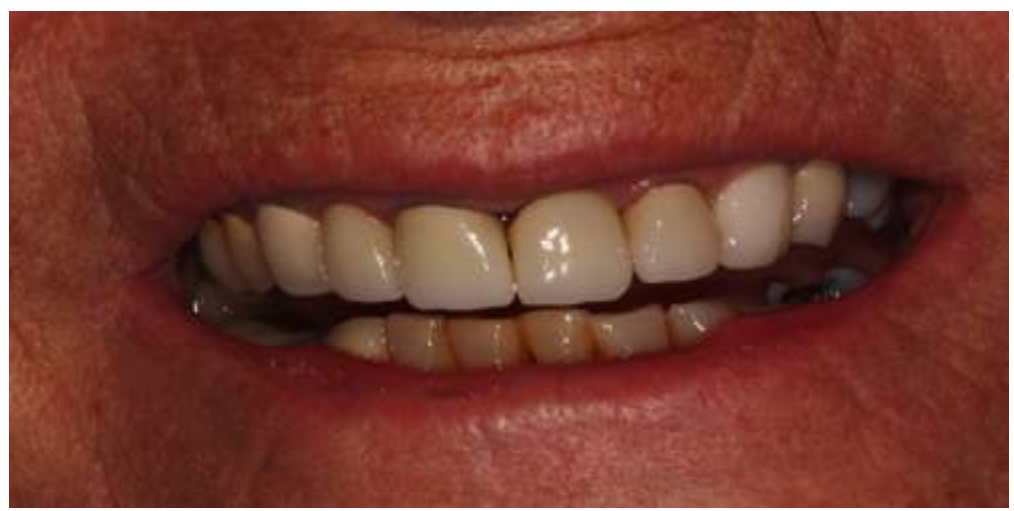

Fig.7 temp re-cemented for 2-3 months

Loading of the implant within $24 \mathrm{~h}$ without functional occlusal contacts provides a superior solution to tackle diffi cult aesthetic problem. The anatomy of the temporary restoration either keeps the original shape of the soft tissue or guides the soft tissue to a correct soft tissue/implant relationship.[20] Various studies have been done to assess success rate of placing implantsin immediate function with unclear results. High survival rate has been reported in various case series of immediate loading of single tooth or short span implants, comparative clinical trials have reported lower survival rates for the same. Chaushu et al. contrasted the surgical results of immediately loaded single-tooth dental implants put into fresh extraction sites to that of instantlyloaded single-tooth implants put into the symptomless site. 19 dental implants had been put into clean extraction sockets, as well as 9 dental implants were positioned directly into recovered sites. They discovered that immediate loading associated with single-tooth dental implants used in fresh extraction sockets had a hazard of a failure estimating to $20 \%$.[21] 
Block et al. identifi ed a substantial distinction within the soft and hard tissue response evaluating immediate along with delayed implant placement following tooth extraction, with quick provisionalization, within maxillary anterior sites; as well as to figure out and also assess the crestal bone levels as being the principal endpoint variable for dental implants positioned and straight away temporized within extraction sockets, to dental implants put into extraction sites once the extraction socket happens to be grafted and healed for 4 months, most promptly restored through an anatomic provisional refurbishment. Within their research, they discovered there was clearly a substantial alteration in the positioning of the facial gingival border, having amuch more apical placement of the facial gingival border within the delayed group in comparison with the immediate group.[22]

Ribeiro evaluated the success rate of maxillary immediate non-functional single-tooth loaded implants immediately placed in extraction socket or healed ridge. No evident diff erences were seen in immediate nonfunctional single-tooth loaded implants in immediate placement conditions in contrast to those inserted under delayed placement condition; both protocols had high success rate in maxillary incisors, canines, and premolars areas.[23] Immediate loading of immediately placed implants in extraction sockets have shown favorable results in maxillary incisors, canines and premolar area. Temporization can be achieved with predictable results when kept out of contact in centric and in excursions. Improved peri implant esthetics areseen as compared to the delayed loading protocol.[24]

The one-piece implant design eliminates the manipulation of the soft tissue portion after initial healing period, therefore the need for placing healing collars or aesthetic abutments is avoided. [25]The immediate construction and placement of provisional restorations after implant insertion, allow for better soft tissue adhesion and seal to form a healthy collar. The restorative process with the one-piece implant resembles that of a natural tooth. The easily prepared abutment part enables an individualized borderline of the preparation to exactly follow the contour of the gingival margin, without violating the soft tissue seal, potentially leading to better preserved interproximal bone and papillae. $[5,7,8]$

The one-piece implants offer a unique simple treatment modality, and have been specially designed for immediate loading of fixed restorations. They are considered an alternative to the conventional implant placement regimen and are ideal for immediate loading in varying bone qualities and quantities. They allow minimally invasive transmucosal flapless placement and limit the requirement for hard tissue grafting procedures.[15,16]

\section{CONCLUSION}

The one-piece implant design provides an attractive and easy alternative to two-piece implants for treatment with immediate provisional restorations. Implant survival rate with beneficial marginal bone levels can be attained with this type of implant design .Clinicians can treat their patients in a highly predictable way if the cases are selected carefully and treatment protocols aimed to achieve the best primary stability are strictly followed. Further studies are needed to determine the long-term success of immediately loaded implants. Furthermore, additional data are needed to determine the minimal bone quality, quantity, minimal insertion torque and maximal occlusal loading for predictable immediate loading protocols

\section{REFERENCES}

[1]. Chen S.T., Buser D. Clinical and esthetic outcomes of implants placed in postextraction sites. Int J Oral Maxillofac Implants. 2009;24 Suppl:186-217.

[2]. Slagter K.W., den Hartog L., Bakker N.A., Vissink A., Meijer H.J., Raghoebar G.M. Immediate placement of dental implants in the esthetic zone: a systematic review and pooled analysis. J Periodontol. 2014;85(7):e241-50.

[3]. Ataullah K, Chee LF, Peng LL, Th o CY, Wei WC, Baig MR. Implant placement in extraction sockets: A short review of the literature and presentation of a series of three cases. J Oral Implantol 2008;34:97-106.

[4]. Abu-Hussein Muhamad,Chlorokostas Georges, Abdulgani Azzaldeen ; Immediate Implants Placed Into Infected Sockets: Clinical Update with 3-Year Follow-Up. . J Dent Med Sci2017,16,1,105-111DOI: 10.9790/0853-160109105111

[5]. Abu-Hussein M, Abdulghani A., Sarafianou A., Kontoes N.; Implants into fresh extraction site: A literature review,case immediate placement report, Journal of Dental Implants. 2013;3(2):160-164

[6]. Bajali M., Abdulgani Azz., Abu-Hussein M, Extraction and immediate implant placement, and provisionalization with two years follow-up: a case report, Int J Dent Health Sci 2014; 1(2): 229236.

[7]. Muhamad et al; Tooth extraction, immediate implant placement: a case report, Asian Pac. J. Health Sci., 2014; 1(4): 543-549

[8]. Abu-Hussein Muhamad Chlorokostas Georges, Abusalih Ahmet, Ismail Hakki Bayraktar, Abdulgani Azzaldeen; Immediate Implant Placement and Loading in Esthetic Zone. Journal of Dental and Medical Sciences 2016 ,1, 71-79,DOI: 10.9790/085315187179

[9]. Schwartz-Arad D, Chaushu G. Placement of implants into fresh extraction sites: 4 to 7 years retrospective evaluation of 95 immediate implants. J Periodontol 1997;68:1110-6.

[10]. Schwartz-Arad D, Gulayev N, Chaushu G. Immediate versus non-immediate implantation for full-arch fi xed reconstruction following extraction of all residual teeth: A retrospective comparative study. J Periodontol 2000;71:923-8.

[11]. Wagenberg BD, Ginsburg TR. Immediate implant placement on removal of the natural tooth: Retrospective analysis of 1,081 implants. Compend Contin Educ Dent 2001;22:399-404, 406, 408

[12]. Nezar Watted, Muhamad Abu-Hussein ;Multidisciplinary Aesthetic Dental Treatment; Peg lateral with Congenitally Maxillary lateral Incisors. Journal of Dental and Medical Sciences 2016, 15, 10, 83-91,DOI: 10.9790/0853-1510018391 
[13]. Mai A, Azzaldeen A, Nezar W, Chlorokostas G, Muhamad AH; Extraction and Immediate Implant Placement with SingleStageSurgical Procedure: Technical Notes and a Case Report. J Dent Med Sci2016, 15: 95-101

[14]. Abu-Hussein M, Watted N, Abdulgani A ;Managing congenitally missing lateral incisors with single tooth implants. Dent Oral Craniofac Res 20162: 318-324

[15]. Drago CJ. Clinical and histological assessment of a one-piece implant system: a pilot study. Dent Prax 2005; 11: 319-325.

[16]. Parel SM, Schow SR. Early clinical experience with a new one piece implants system in single tooth sites.JOral Maxillofac Surg2005; 63:2 -10.

[17]. Abu-Hussein M, Watted N, Shamir D ;A Retrospective Study of the AL Technology Implant System used for Single-Tooth Replacement. Int J Oral Craniofac Sci 2016,2(1): 039-046. DOI: 10.17352/2455-4634.000017

[18]. Muhamad AH, Azzaldeen A, Nezar W, Mohammed Z. ; Esthetic Evaluation of Implants Placed after Orthodontic Treatment in Patients with Congenitally Missing Lateral Incisors. J Adv Med Dent Scie Res2015 ;3(3):110-118

[19]. Abu-Hussein M, Georges C, Watted N, Azzaldeen A ;A Clinical Study Resonance Frequency Analysis of Stability during the Healing Period. Int J Oral Craniofac Sci 2016,2(1): 065-071. DOI: 10.17352/2455-4634.000021

[20]. Tsirlis AT. Clinical evaluation of immediate loaded upper anterior single implants. Implant Dent 2005;14:94-103.

[21]. Chaushu G, Chaushu S, Tzohar A, Dayan D. Immediate loading of single-tooth implants: Immediate versus non-immediate implantation. A clinical report. Int J Oral Maxillofac Implants 2001;16:267-72.

[22]. Block MS, Mercante DE, Lirette D, Mohamed W, Ryser M, Castellon P. Prospective evaluation of immediate and delayed provisional single tooth restorations. J Oral Maxillofac Surg 2009;67:89-107.

[23]. Ribeiro FS, Pontes AE, Marcantonio E, Piattelli A, Neto RJ, Marcantonio E Jr. Success rate of immediate nonfunctional loaded single-tooth implants: Immediate versus delayed implantation. Implant Dent 2008;17:109-17.

[24]. Tarnow DP, Emtiaz Classi A. Immediate loading of threaded implants al stage 1 surgery in edentulous arches: ten consecutive case reports with 1- to 5-year data.Int J Oral Maxiiiofac implants 1997;12: 319-324

[25]. Chen ST, Wilson TG Jr, Hommerle CH. Immediate or early placement of implants following tooth extraction: Review of biologic basis, clinical procedures, and outcomes. Int J OralMaxillofac Implants 2004; 19: 12-25. 